\title{
Correlation of embedded nanowires probed by x-ray off-Bragg scattering of the host matrix
}

\author{
Thomas Tran, ${ }^{a}$ Xiaorong Weng, ${ }^{a \llbracket l}$ Marcel Hennes, ${ }^{a}{ }^{\ddagger}$ Dominique Demaille, ${ }^{a}$ Alessan-dro Coati, ${ }^{b}$ Alina Vlad, ${ }^{b}$ Yves \\ Garreau, ${ }^{b, c}$ Michèle Sauvage-Simkin, ${ }^{b}$ Maurizio Sacchi, ${ }^{a, b}$ Franck Vidal ${ }^{a}$ and Yunlin Zheng ${ }^{a}$
}

We show that information on the spatial correlation of nano-objects embedded in a crystalline matrix can be retrieved by analyzing the x-ray scattering around Bragg reflections of the host ma-trix. We report data for vertically aligned $\mathrm{Ni}$ and CoNi-alloy nanowires (NWs) in a $\mathrm{SrTiO}_{3}$ matrix. When the Bragg condition is fulfilled for the matrix and not for the NWs, the latter can be approxi-mated by voids and the scattering around the matrix reflections contains information on the NWs self-correlation (i.e., on their diameter $d$ ) and on the correlation between NWs (interdistance $D$ ). Non-destructive synchrotron x-ray diffraction data provide information on these values averaged over large areas, complementing local transmission electron microscopy observations. Our mea-surements show that the off-Bragg scattering around the matrix reflections can be exploited to study a wide range of embedded nano-objects, with no constraint on their crystallinity, strain or presence of defects.

\section{Introduction}

Nanostructured composites open up many perspectives, such as obtaining artificial multiferroic or multifunctional materials by coupling properties of their components (Chen \& Jia, 2021; Chen et al., 2019; Sun et al., 2019). Vertically aligned nanocomposites are particularly interesting in this context (Hennes et al., 2021; Huang et al., 2021), given a large interface of interaction between the components on the one hand and the possibility of stacking nanostructured layers on the other hand (Kim et al., 2014). Examples of complex structures include, for instance, vertically aligned $\mathrm{ZnO}$ nanowires (NWs) capped by $\mathrm{Au}$ nanoparticles embedded in a piezoelectric $\mathrm{BaTiO}_{3}$ matrix for optical property tuning (Misra et al., 2020) and Ag nanorods on top of Co particles and capped by Co nanoclusters in a $\mathrm{TiO}_{2}$ matrix for photo-catalysis (Watanabe et al., 2014). Further axial structuration along NWs can be envisaged, for instance in the perspective of ultrafast photo-assisted magnetization switching in ferro-

\footnotetext{
${ }^{a}$ Sorbonne Université, CNRS-UMR 7588, Institut des Nanosciences de Paris (INSP), F75005 Paris, France

${ }^{b}$ Synchrotron Soleil, L'Orme des Merisiers Saint-Aubin BP 48, 91192 Gif-sur-Yvette Cedex, France

'Université Paris Diderot, Sorbonne Paris Cité, CNRS-UMR 7162, Laboratoire Matériaux et Phénomènes Quantiques (MPQ), 75205 Paris Cedex 13, France

IPresent address: Université Grenoble Alpes, CEA, IRIG/DePhy/MEM/NRS, 38054 Grenoble, France

*Present address: Sorbonne Université, CNRS-UMR 7614, Laboratoire de Chimie Physique - Matière et Rayonnement (LCPMR), F-75005 Paris, France
}

magnetic NWs capped by plasmonic nanoclusters (Ikemiya et al., 2014; Xu et al., 2015; Pancaldi et al., 2019).

The complexification of multicomponent matrix/inclusions structures leads to an increasing need for investigation tools that complement local observation means, which are often destructive. No versatile and non-destructive analysis method exists for probing the morphology of nano-objects buried in a layer. Small angle x-ray scattering (SAXS) is a powerful technique to analyze nano-objects on or in a soft matter layer, for instance membranesupported nanoparticles (Li et al., 2016). When a conventional substrate is involved, the SAXS transmission geometry requires delicate and non-damaging techniques to remove the substrate first (Wong et al., 1998; Bourlier et al., 2020; Takahashi, R. \& Lippmaa, 2020), in order to obtain a free-standing ultrathin film containing the nano-objects. Grazing incidence SAXS (GISAXS) is a versatile tool for nano-objects on a surface (Renaud et al., 2009). Buried objects contribute an extremely weak GISAXS signal with respect to the strong scattering from the surface and from the embedding layer. Therefore, its analysis is complex (Lazzari, 2002) and almost impossible, e.g. when the objects and the layer have similar mass densities or when the layer of interest is capped by other layers and structures. Here, we propose to use a Bragg reflection being sensitive only to the matrix layer of interest and to analyze the scattering induced by objects buried into it, a general non-destructive method that can be applied whenever the matrix is crystalline. 


\section{Experimental details}

$\mathrm{Ni}$ and CoNi-alloy NWs were embedded in a $\mathrm{SrTiO}_{3}$ (STO) matrix on STO(001) substrates by sequential pulsed laser deposition, using procedures already described in previous works (Bonilla et al., 2013; Schuler et al., 2016; Hennes et al., 2018). A quadrupled Nd:YAG laser (wavelength $266 \mathrm{~nm}$ ) operating at $10 \mathrm{~Hz}$ with a fluence in the 1-3 ${\mathrm{J} . \mathrm{cm}^{-2}}^{-2}$ range was used. The pressure during the growth was of the order of $10^{-6} \mathrm{mbar}$ and the temperature of the substrate was $650^{\circ} \mathrm{C}$. The growth procedure consists in repeating a basic sequence of laser shots, alternately on $\mathrm{STO}, \mathrm{NiO}$ and $\mathrm{CoO}$ targets. Each sequence deposited about $0.1 \mathrm{~nm}$ of STO matrix and $0.01 \mathrm{~nm}$ of $\mathrm{Ni}$ or Co. The ratio of the numbers of shots on the STO target and on the NiO-CoO targets in the basic sequence determines the NW diameter and their volume fraction in the epilayer. In the case of CoNi-alloy NWs, the Co content in the NWs is varied by changing the ratio of the shots on the $\mathrm{CoO}$ and $\mathrm{NiO}$ targets.

The structure of the samples was studied by transmission electron microscopy (TEM) and x-ray diffraction (XRD). TEM images were recorded using a JEOL JEM 2100F microscope equipped with a field-emission gun operated at $200 \mathrm{kV}$ and a Gatan GIF spectrometer. The alloy composition was measured by $\mathrm{x}$-ray energy dispersive spectroscopy. Laboratory XRD measurements were made on a 5-circle diffractometer (Rigaku SmartLab) using a channel-cut $2 \times \mathrm{Ge}(220)$ monochromator to select the $\mathrm{Cu} \mathrm{K} \alpha 1$ radiation from a rotating anode. Synchrotron XRD and grazing incidence XRD (GIXRD) measurements were carried out at the SixS beamline of the SOLEIL synchrotron, using either $15.6 \mathrm{keV}$ or $18.4 \mathrm{keV}$ photon energies. Data were acquired in a continuous mode with a 2D detector (XPAD), to efficiently scan a wide volume of reciprocal space (Li et al., 2020). Except for specular reflections, reciprocal space maps of all other reflections were collected at $0.3^{\circ}$ grazing incidence (GIXRD). Data were processed using the Binoculars software (Roobol et al., 2015). In the following, all reciprocal coordinates are expressed in the reciprocal lattice units (r.l.u.) of bulk STO with the lattice parameter of 0.3905 nm.

\section{Results and analysis}

\subsection{Samples}

The three samples of $\mathrm{Ni}$ and CoNi NWs analyzed are listed in Table 1 The NWs were grown with a high aspect ratio length / diameter $(t / d)$ for shaping their magnetic properties. Sample $C$ is composed of CoNi NWs ( $t=59 \mathrm{~nm})$ which are capped by a 5 $\mathrm{nm}$ thick Au:STO layer with a Au volume fraction of $10 \%$. NW typical sizes and densities are shown in TEM plan-view images of samples A and B (Fig 1). The inset in Figure 1(b) displays a TEM cross-section view of sample $B$ where the presence of NWs is revealed by moiré fringes due to the superposition of NWs and the matrix along the electron path. NWs have a roughly round shape without visible facets. Mean diameters of NWs are in the range of 3.2-5.7 $\mathrm{nm}$, with a relative standard deviation of about $10 \%$. Previous studies on Ni NWs (Weng et al., 2018) and on CoNi NWs (Weng et al., 2020) established that NWs are epitaxied cube-oncube in the matrix. The NW diffraction spots have a complex and widespread structure that reflects locally varying strain and tilt along the NWs. Under these conditions, the extension of the NW spots does not match the NW form factor.

Table 1 Sample list and mean characteristics. $a_{\mathrm{z}}^{\text {mat }}$, matrix out-of-plane lattice parameter determined by XRD; $t$, film thickness measured by XRD and observation in TEM cross-section; $n$ and $d$, NW number density and diameter respectively, determined from a statistic over tens to hundreds of NWs in TEM plan-view.

\begin{tabular}{cccccc}
\hline & $\mathrm{NW}$ type & $a_{\mathrm{z}}^{\text {mat }}(\mathrm{nm})$ & $t(\mathrm{~nm})$ & $n\left(\mathrm{~cm}^{-2}\right)$ & $d(\mathrm{~nm})$ \\
\hline $\mathrm{A}$ & $\mathrm{Ni}$ & 0.3942 & 51 & $4.1 \times 10^{11}$ & 5.7 \\
$\mathrm{~B}$ & $\mathrm{Ni}$ & 0.3931 & 231 & $18 \times 10^{11}$ & 4.1 \\
$\mathrm{C}$ & $\mathrm{Co}_{0.8} \mathrm{Ni}_{0.2}$ & 0.3957 & 59 & $13 \times 10^{11}$ & 3.2 \\
\hline
\end{tabular}
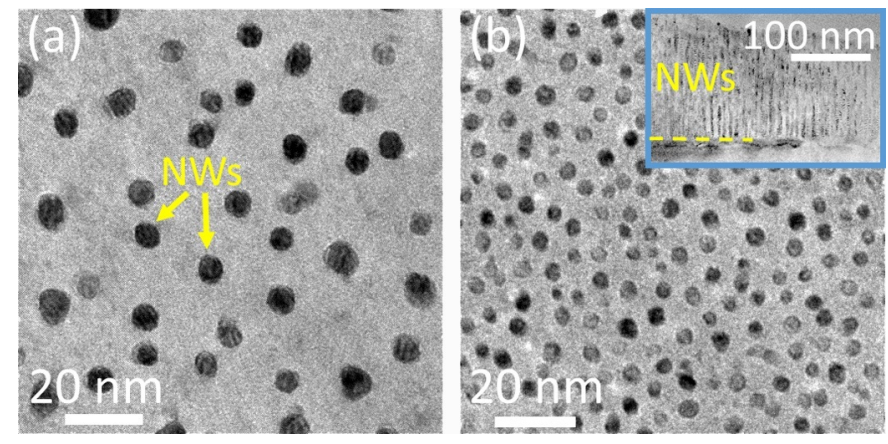

Fig. 1 TEM plan-view of samples A (a) and B (b). Inset in (b): crosssection view of sample $B$ where the yellow dashed line indicates the interface between the epilayer and the substrate.

The matrix is epitaxial on the substrate, with an in-plane (IP) lattice parameter close to the STO bulk one and an out-of-plane (OP) lattice expansion of $0.7-1.3 \%$ (Tab 1). The matrix lattice expansion is due to the oxygen-free condition used during growth, which is needed to preserve the metallic character of NWs. In all the samples, a laboratory XRD $\theta / 2 \theta$ scan displayed a well-defined 002 Bragg peak of the matrix and associated oscillations. The peak full-width at half maximum (FWHM) and the oscillation period correspond to the inverse of the matrix thickness measured by TEM, indicating a full vertical coherency of the matrix. The rocking curves of the matrix 002 reflection in all samples contain a diffuse part extending to several degrees. If the extension of the rocking curves was interpreted as the IP coherency of the matrix, the coherent length would drop to a few nm only, which would be intriguing and problematic for a single crystalline matrix. We will address in detail this last point by synchrotron XRD measurements.

\subsection{XRD measurements}

Figure 2 sketches the arrangement of diffraction spots of the substrate, the matrix and NWs. The spots of epitaxial $f c c \mathrm{Ni}$ and CoNi NWs are localized near the strong reflections of STO, which are called here fcc-compatible and involve in their structure factors the constructive contribution of $\mathrm{Sr}$ and $\mathrm{O}$ atomic diffusion 


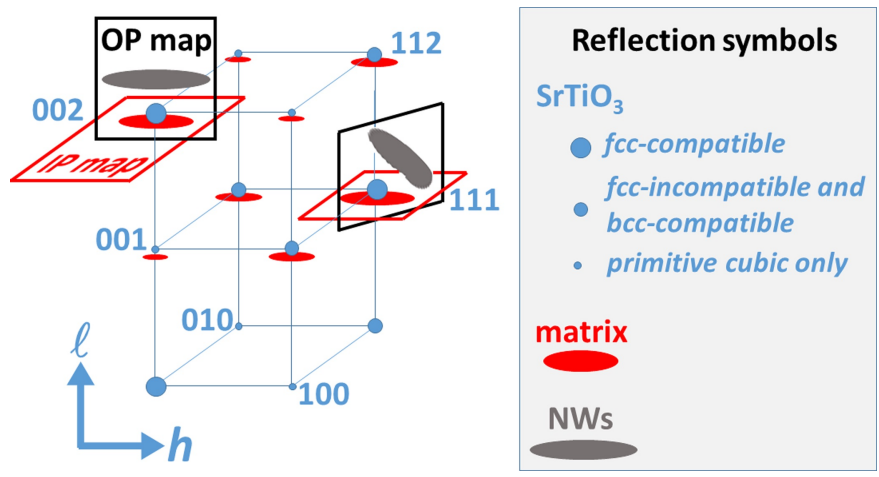

Fig. 2 Reciprocal space sketch. The STO reflections are classified into three types and symbolized by different spot sizes. An OP map of a reflection (black rectangles) corresponds to a vertical and IP radial cut. An IP map (red rectangles) is a horizontal cut at the matrix spot position.

at least: $F_{h k l}=f_{\mathrm{Sr}} \pm f_{\mathrm{Ti}}+3 f_{\mathrm{O}}$ ( + or - for even or odd $h k l$, respectively). Medium strength reflections, such as 112 , concern an in-phase contribution of $\mathrm{Sr}$ and $\mathrm{Ti}$ with an anti-phase one of $\mathrm{O}\left(F_{h k l}=f_{\mathrm{Sr}}+f_{\mathrm{Ti}}-f_{\mathrm{O}}\right)$, while weak reflections involve an anti-phase contribution of $\mathrm{Ti}$ and $\mathrm{O}$ with respect to that of $\mathrm{Sr}$ $\left(F_{h k l}=f_{\mathrm{Sr}}-f_{\mathrm{Ti}}-f_{\mathrm{O}}\right)$.
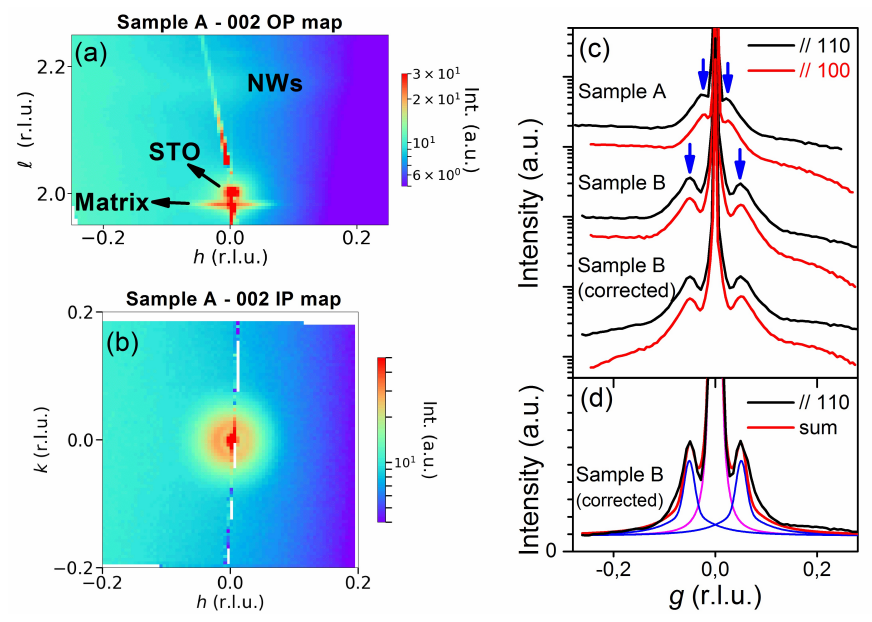

Fig. 3002 maps and profiles. (a) OP map of sample A. The inclined diffuse line is due to the substrate. The map intensity is displayed on a logarithmic scale. (b) IP map of sample A. (c) IP profiles of samples A and $B$ along 110 (black) and 100 (red) directions, with the beam footprint correction made for the curves of sample B. $g$ coordinates are relative to the spot center. The blue arrows outline the position of the scattering ring. The profile intensity is on a logarithmic scale. (d) Decomposition of the 110 corrected profile (black) of sample B into one central (magenta) and two secondary (blue) peaks, as well as their sum (red). The profile intensity is on a linear scale.

In all samples, the 002 specular reflection of the matrix is beneath that of the substrate, as a consequence of the matrix OP lattice expansion (Fig 3(a)). The spot degenerates into a horizontal diffuse line in the OP map, reflecting the existence of vertical objects inside the matrix. By examining the IP structure of the spot, a scattering ring was evidenced (Fig $3(\mathrm{~b})$ ). A decreasing intensity slope is observed in the maps from negative to positive $h$ coordi- nates, due to the irradiated area that changes during a specular scan. The normalization of the intensity by the incident beam footprint on the sample corrects the slope (Fig 3 (c-d)). The radius of the scattering ring $g_{1}$ is equal to 0.023 and 0.051 r.l.u. for samples A and B corresponding to a length of 17.0 and $7.7 \mathrm{~nm}$, respectively. These values do not correspond to the NW diameter $d$, but could be related to NWs' interdistance $D$. If a hexagonal arrangement of the NWs is assumed, an estimate of their mean interdistrace can be made from the NW density determined by TEM (Tab 1): $D^{\text {TEM }}=16.8$ and $8.0 \mathrm{~nm}$ for samples A and B, respectively. One notices that beyond the ring, the intensity does not drop to noise, indicating additional information contained in the diffuse signal.
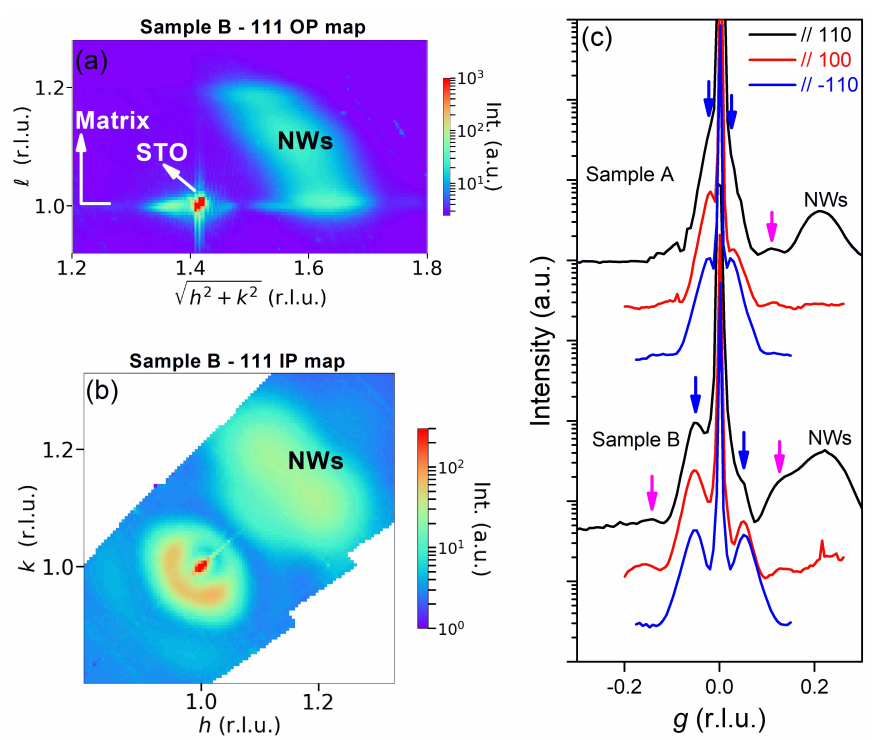

Fig. 4111 maps and profiles. (a) OP map of sample B. (b) IP map of sample B. (c) IP profiles of samples A and B along 110, 100 and $\overline{1} 10$ directions. The blue arrows outline the position of the first scattering ring and the magenta ones the position of the second ring.

Non-specular measurements were made in GIXRD mode under a constant grazing incidence of $0.3^{\circ}$, approximately twice the STO critical angle. This ensures a constant and large irradiated area of the samples during the scans, and increases the relative epilayerto-substrate sensitivity. Once again, scattering from the matrix occurs in the horizontal plane (Fig 4) with a ring similar to that observed around the 002 specular spot. An intensity asymmetry is observed along the radial 110 direction, shifting slightly the apparent ring radial positions with respect to the transverse ones along the $\overline{1} 10$ direction. As the same shape is observed in different reflections, the scattering ring does not reflect a texture of the matrix, but rather constitutes a satellite of the Bragg spot, resulting from a convolution in reciprocal space as for a form factor. Interestingly, a faint second ring was also observed. Along the radial direction, it is overlapped by the broad spot of the NWs. The radius $g_{2}$ of the second ring is around 0.109 and 0.135 r.l.u., or the inverse of 3.6 and $2.9 \mathrm{~nm}$, for samples A and B respectively. These lengths do not correspond directly to the expected NWs' diameter or interdistance. In addition, the second ring cannot be 
understood as a harmonic of the first one, since their radius ratio, 4.7 and 2.6 respectively for samples A and B, does not follow the harmonicity in a cylindrical symmetry problem often associated to Bessel functions.

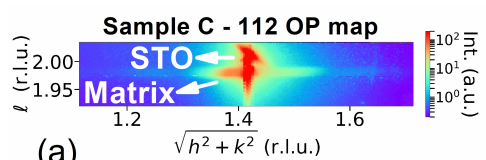

(a)
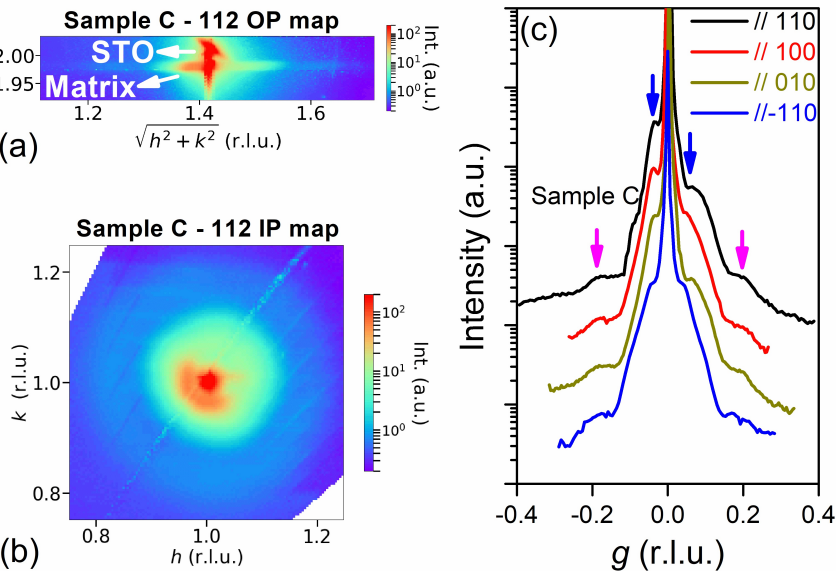

Fig. 5112 maps and profiles (sample C). (a) OP map. (b) IP map. (c) IP profiles along $110,010,010$ and $\overline{1} 10$ directions. The blue arrows outline the position of the first scattering ring and the magenta ones the position of the second ring.

The use of the medium strength STO 112 reflection, forbidden for $f c c$, removed the contribution of NWs and enhanced the diffuse signal of the matrix, as illustrated by the maps of sample $\mathrm{C}$ (Fig 5). The radius of the first ring is 0.040 r.l.u., or the inverse of $9.8 \mathrm{~nm}$, and could be linked to the NW interdistance of $9.4 \mathrm{~nm}$ in sample $\mathrm{C}$ evaluated by TEM. The second ring is 4.9 times larger than the first one and has not a direct interpretation yet. A similar radial asymmetry in intensity of the rings is persistent. Data for the other medium strength reflection 301 confirmed that the asymmetry is always radial and not related in particular to the 110 direction involved in 111 and 112 reflections.

\subsection{Analysis}

When nano-objects have a crystal lattice different from that of the host matrix, they do not contribute to a Bragg reflection of the matrix and can be considered as voids or holes. XRD measurements are thus comparable to those of porous materials (Buttard et al., 2002; Punegov et al., 2007). A general form function of holes $f(\mathbf{r})$ is defined as 1 inside the holes and 0 elsewhere. The electronic density of the matrix $\rho(\mathbf{r})$ can be seen as that of a virtual continuous film $\rho^{\text {film }}(\mathbf{r})$ multiplied by $1-f(\mathbf{r})$. The Fourier transform (FT) of $\rho(\mathbf{r})$ is then: $\rho(\mathbf{q})=\rho^{\text {film }}(\mathbf{q})-\rho^{\text {film }}(\mathbf{q}) \otimes f(\mathbf{q})$, where $\otimes$ represents the convolution and $\mathbf{q}$ a scattering vector in the vicinity of a Bragg one $\mathbf{q}_{\mathrm{B}}$. For a narrow film Bragg reflection, the scattering intensity $I\left(\mathbf{q} \neq \mathbf{q}_{\mathrm{B}}\right)$ is only due to the form and proportional to $\left|\rho^{\text {film }}\left(\mathbf{q}_{\mathrm{B}}\right)\right|^{2} \times\left|f\left(\mathbf{q}-\mathbf{q}_{\mathrm{B}}\right)\right|^{2}$. This means that the scattering is equivalent to that from virtual nano-objects made of matrix matter instead of holes, i.e. as for the negative pattern of a porous matrix.

The intensity due to the general form of holes $|f(\mathbf{q})|^{2}$ accounts for the squared modulus of the form factors of all the holes and the interference terms. This is simplified in the ideal case of homogeneous holes and the form function becomes $f(\mathbf{r})=$ $f_{\mathrm{h}}(\mathbf{r}) \otimes f_{\mathrm{p}}(\mathbf{r})$, where $f_{\mathrm{h}}(\mathbf{r})$ is the form function of one hole and $f_{\mathrm{p}}(\mathbf{r})$ the hole positions. It follows that the scattering intensity is given by a product of the squared modulus of one hole form factor $\left|f_{\mathrm{h}}(\mathbf{q})\right|^{2}$, i.e. Airy pattern here, and the FT of the self-correlation of hole positions $\left|f_{\mathrm{p}}(\mathbf{q})\right|^{2}$. The self-correlation of hole positions $f_{\mathrm{p}}(\mathbf{r}) \otimes f_{\mathrm{p}}(\mathbf{r})$ can be normalized and expressed in terms of the hole positional pair function $p(\mathbf{r})$ as: $\delta(\mathbf{r})+n \times p(\mathbf{r})$, where $n$ is the hole density. The associated intensity, $I_{\mathrm{p}}(\mathbf{q})=\left|f_{\mathrm{p}}(\mathbf{q})\right|^{2}$, is linked to the pair function $p(\mathbf{r})$ by: $I_{\mathrm{p}}(\mathbf{q}) \propto 1+n \times p(\mathbf{q})$.

Pair functions are widely used to describe a short, mean or long-range order in matter, from gas and liquid to solid-state phases (Hosemann \& Bagchi, 1952; Guinier, 1963). Following the concept of paracrystal developed by Hosemann and Bagchi (1962), a short-order 1D or 2D system can be described by the nearest-neighbor distance $D$ and its variance $\sigma^{2}$, the variance being increasing and cumulative for next neighbors. The scattering intensity in the $1 \mathrm{D}$ case is then:

$$
I_{\mathrm{p}}(q)=\left\{1-G^{2}(q)\right\} /\left\{1-2 \times \cos (q D) \times G(q)+G^{2}(q)\right\}
$$

where $G(q)=\exp \left(-q^{2} \sigma^{2} / 2\right)$ is the FT of the Gaussian distribution of the first neighbors.
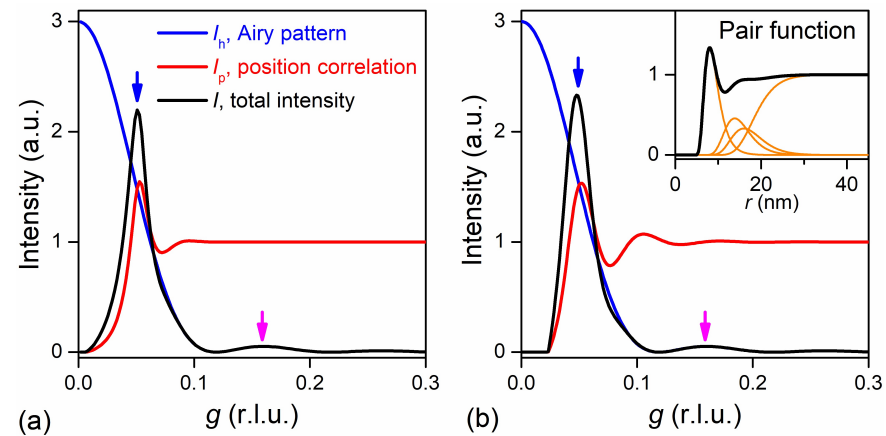

Fig. 6 Scattering profiles, calculated with $D=8, \sigma=2$ and $d=4 \mathrm{~nm}$, showing the intensity due to the form $I_{\mathrm{h}}$ (blue), that due to the position correlation $I_{\mathrm{p}}$ (red) and the total intensity $I$ (black). The blue and magenta arrows outline the first and second scattering rings, respectively. (a) Profiles calculated according to the paracrystal order of neighbors. (b) Profiles calculated using asymmetric distributions of neighbors. Inset: the resulting pair function (black) and the contributions of the three first neighbors as well as that of all other neighbors (orange).

For our vertically aligned NWs, we analyze the horizontal scattering as a function of $\Delta \mathbf{q}_{\|}$, the scattering vector IP deviation from a Bragg position: $\Delta \mathbf{q}_{\|}=\mathbf{q}-\mathbf{q}_{\mathrm{B}}$ or $2 \pi \mathbf{g}$ with $g$ in r.l.u.. The generalization of Equation 1 to the 2D case (Eads \& Millane, 2001), together with the hole form factor of diameter $d$, allows us to reproduce qualitatively the positions of the scattering rings measured in our samples (Fig6(a)). A more realistic picture should include a cut-off at short distances $(<d)$ in the distribution of first neighbors. We implemented this by considering a hexagonal arrangement of non-overlapping holes disordered by a log-normal distribution of the first neighbors and by an increasing disorder for next neighbors. The results obtained using the parameters of 
Table 2 NW mean separation and diameter determined by the scattering rings. $g_{1}$ and $\Gamma_{1}$ : measured radius and standard deviation of the first ring; $g_{2}$ : measured radius of the second ring; $D$ and $\sigma$ : calculated NW interdistance and its deviation; $d$, calculated NW diameter.

\begin{tabular}{ccccccc}
\hline & $\begin{array}{c}g_{1} \\
\text { (r.l.u.) }\end{array}$ & $\begin{array}{c}\Gamma_{1} \\
\text { (r.l.u.) }\end{array}$ & $\begin{array}{c}g_{2} \\
\text { (r.l.u.) }\end{array}$ & $\begin{array}{c}D \\
(\mathrm{~nm})\end{array}$ & $\begin{array}{c}\sigma \\
(\mathrm{nm})\end{array}$ & $\begin{array}{c}d \\
(\mathrm{~nm})\end{array}$ \\
\hline A & 0.023 & 0.008 & 0.109 & 16.2 & 5.9 & 5.9 \\
B & 0.051 & 0.010 & 0.135 & 7.5 & 1.8 & 4.7 \\
C & 0.040 & 0.012 & 0.194 & 9.7 & 3.0 & 3.3 \\
\hline
\end{tabular}

Table 2 are shown in Figure 6(b)). The first ring radius is related to the NW separation. The derived $D$ value is slightly different from the inverse of $g_{1}$, since the ring is on the slope due to the form, i.e. the Airy disc, and the 2D problem is concerned here. The relative deviation of the first neighbors $\sigma / D$ was evaluated to be around $30 \%$. Interestingly, a second ring is detected that can be understood as the first ring of the form factor, i.e. the first secondary maximum of the Airy pattern, with $g_{2} \approx 1.635 / d$. The deduced values of the NW diameter $d$ (Tab.2) are in agreement with those determined by TEM (Tab 1). It should be noted that GIXRD probes an area of several $\mathrm{mm}^{2}$, averaging $d$ over more than $10^{10}$ NWs.

\subsection{Discussion}

We discuss and examine the measurement efficiency and the calculation relevance, providing complementary elements that have not addressed before. In grazing $h k 0$ reflections (200, 400, 220, 110 and 100), the matrix and substrate spots are superposed due to the IP pseudomorphism in the homoepitaxy and the weak diffuse signal was not observed. The 002 specular reflection allowed to resolve the first scattering ring. The use of the harmonic, 004 reflection, did not improve the signal. The grazing incidence for non-specular reflections, 111, 202 and 113, decreased the substrate influence and increased the ring visibility. Furthermore, 112 and $301 f c c$ forbidden reflections removed contributions from the NWs and enhanced the signal contrast. A radial asymmetry in scattering is observed for non-specular reflections. No intrinsic reason was found for this effect. Although we have no sound explanation yet, this effect can reasonably be ascribed to the presence of strong reflections from the substrate, which in our systems are always in close proximity. It is to be noticed that in a coherent XRD experiment on a single free-standing GaAs nanorod grown on GaAs substrate (Biermanns et al., 2009), the symmetrical form pattern of the nanorod also seems perturbed by the close substrate spot. To elucidate the question on the radial asymmetry in non-specular reflections, it would be instructive and interesting to investigate a case where the matrix lattice is sufficiently different from that of the substrate.

In order to be able to single out the hole contribution to the scattering and to model it as a separate form factor, the matrix Bragg reflections must be narrow compared to the rings generated by the embedded nanostructures. This condition is fulfilled in our case since the IP width of the matrix Bragg peaks is at least ten times smaller than the widths of the scattering rings associated with the NWs. Also, the assumption of homogeneous holes allows one to simplify the description. In a heterogeneous assembly of holes, large numerical simulation would be required. In our case, a standard deviation of about $10 \%$ in NW diameter has little effect on the slope of the form factor and then the first scattering ring. The position and the width of the second ring are determined mainly by the NW mean diameter. A diameter dispersion should increase the width and reduce the intensity of the second ring, and impact even more drastically the form factor rings of higher order.

The present study concerned close packed NWs of short-range order. Additional scattering rings related to the position correlation are expected in a more diluted $(D / d \gg 1)$ and ordered system. This would provide information on the self-assembly mechanisms of nano-objects and on the order-disorder state of their organization.

\section{Conclusions}

Nano-objects embedded in a matrix can be considered as holes for a matrix Bragg reflection. Off-Bragg scattering is equivalent to that from virtual nano-objects made of matrix matter alone. The first scattering ring results from the position correlation of nano-objects and from their form. The position and the width of the ring provide the interdistance of nano-objects and its variance. For a short-range order system, only the form factor intervenes further and the second scattering ring corresponds to the first ring of the form factor. This study points out the selectivity and sensitivity of XRD for probing nano-objects by scattering around a Bragg reflection of the host matrix. This calls for the use of off-Bragg scattering to study nano-objects inside a particular crystalline structure through nanocomposites and stacked nanostructures, in a non-destructive way.

\section{Acknowledgements}

X.W. acknowledges financial support of the Chinese Government Scholarship from China Scholarship Council (CSC No. 201608070062). M.H. acknowledges financial support from the French Embassy in Berlin (Service pour la Science et la Technologie) and Campus France. Dr. A. Resta of the SOLEIL SixS beamline is kindly acknowledged for introducing us to the use of the Binoculars software and XRD data processing. The authors gratefully thank F. Breton for his assistance and design of the PLD control system, IMPMC (CNRS-Sorbonne Université) for access to the TEM facilities and Dr. J.-M. Guigner for his help. This work has been funded in part by the LabEx Matisse (French projects ANR-11-IDEX-0004-02/10-LABX-0067-MATISSE). We acknowledge SOLEIL for provision of synchrotron radiation facilities through the proposals $n^{\circ} 20160182$ and $n^{\circ} 20200524$.

\section{References}

1 Biermanns, A., Davydok, A., Paetzelt, H., Diaz, A., Gottschalch, V., Metzgerd, T. H. \& Pietscha, U. (2009). J. Synchrotron Rad. 16, 796-802.

2 Bonilla, F. J., Novikova, A., Vidal, F., Zheng, Y., Fonda, E., 
Demaille, D., Schuler, V., Coati, A., Vlad, A., Garreau, Y., Sauvage-Simkin, M., Dumont, Y., Hidki, S. \& Etgens, V. H. (2013). ACS Nano 7, 4022-4029.

3 Bourlier, Y., Bérini, B., Frégnaux, M., Fouchet, A., Aureau, D. \& Dumont, Y. (2020). ACS Appl. Mater. Interfaces 12, 84668474.

4 Buttard, D., Bellet, D., Dolino, G. \& Baumbach, T. (2002). J. Appl. Phys. 91, 2742-2752.

5 Chen, A. \& Jia, Q. (2021). MRS Bulletin 46, 115-122.

6 Chen, A., Su, Q., Han, H., Enriquez, E. \& Jia, Q. (2019). Adv. Mater. 31, 1803241.

7 Eads, J. L. \& Millane, R. P. (2001). Acta. Cryst. A 57, 507-517.

8 Hennes, M., Demaille, D., Patriarche, G., Tran, T., Zheng Y. \& Vidal, F. (2021). MRS Bulletin 46, 136-141.

9 Hennes, M., Schuler, V., Weng, X., Buchwald, J., Demaille, D., Zheng, Y. \& Vidal, F. (2018). Nanoscale 10, 7666-7675.

10 Guinier, A. (1963). X-ray Diffraction in Crystals, Imperfect Crystals, and Amorphous Bodies, Dover Publications, Inc, New York.

11 Hosemann, R. \& Bagchi, S. N. (1952). Acta Cryst. 5, 612-614.

12 Hosemann, R. \& Bagchi, S. N. (1962). Direct Analysis of Diffraction by Matter, Amsterdam: North-Holland.

13 Huang, J., Li, W., Yang, H. \& MacManus-Driscoll, J. L. (2021). MRS Bulletin 46, 159-167.

14 Ikemiya, K., Konishi, K., Fujii, E., Kogure, T., KuwataGonokami, M. \& Hasegawa, T. (2014) Opt. Mater. Express 4, 1564-1573.

15 Kim, D. H., Aimon, N. M., Sun, X. \& Ross, C. A. (2014). Adv. Funct. Mater. 24, 2334-2342.

16 Lazzari, R. (2002). J. Appl. Cryst. 35, 406-421.

17 Li, N., Dupraz, M., Wu, L., Leake, S. J., Resta, A., Carnis, J., Labat, S., Almog, E., Rabkin, E., Favre-Nicolin, V., Picca, F.E., Berenguer, F., van de Poll, R., Hofmann, J. P., Vlad, A.,
Thomas, O., Garreau, Y., Coati, A. \& Richard, M.-I. (2020). Sci. Rep. 10, 12760.

18 Li, T, Senesi, A. J. \& Lee, B. (2016). Chem. Rev. 116, 11128 11180.

19 Misra, S., Zhang, D., Qi, Z., Li, D., Lu, J., Chen, H.-T. \& Wang, H. (2020). Cryst. Growth Des. 20, 6101-6108.

20 Pancaldi , M., Leo, N. \& Vavassori, P. (2019). Nanoscale 11, 7656-7666.

21 Punegov, V. I., Lomov, A.A. \& Shcherbachev, K. D. (2007). phys. stat. sol. (a) 204, 2620-2625.

22 Renaud, G., Lazzari, R. \& Leroy, F. (2009). Surf. Sci. Rep. 64, 255-380.

23 Roobol, S., Onderwaater, W., Drnec, J., Felici, R. \& Frenken, J. (2015). J. Appl. Cryst. 48, 1324-1329.

24 Schuler, V., Milano, J., Coati, A., Vlad, A., Sauvage-Simkin, M., Garreau, Y., Demaille, D., Hidki, S., Novikova, A., Fonda, E., Zheng, Y. \& Vidal, F. (2016). Nanotechnology 27, 495601.

25 Sun, X., Li, Q., Huang, J., Jian, J., Lu, P., Zhang, X., MacManus-Driscoll J. L. \& Wang, H. (2019). J. Appl. Phys. 125, 082530.

26 Takahashi, R. \& Lippmaa, M. (2020). ACS Appl. Mater. Interfaces 12, 25042-25049.

27 Watanabe, A., Kotake, Y., Kamata, Y., Chikamatsu, A., Ueno, K., Misawa, H. \& Hasegawa, T. (2014). J. Phys. Chem. Lett. 5, 25-29.

28 Weng, X., Hennes, M., Coati, A., Vlad, A., Garreau, Y., Sauvage-Simkin, M., Fonda, E., Patriarche, G., Demaille, D., Vidal, F. \& Zheng, Y. (2018). Phys. Rev. Materials 2, 106003.

29 Weng, X., Hennes, M., Tran, T., Casaretto, N., Demaille, D., Vidal, F. \& Zheng, Y. (2020). CrystEngComm 22, 4730-4739.

30 Wong, W.S., Sands, T. \& Cheung, N. W. (1998). Appl. Phys. Lett. 72, 599-601.

31 Xu, H., Hajisalem, G., Steeves, G. M., Gordon, R. \&Choi, B. C. (2015). Sci. Rep. 5, 15933. 